\title{
Sequences containing no 3-term arithmetic progressions
}

\author{
Janusz Dybizbański \\ Institute of Informatics \\ University of Gdańsk, Poland \\ jdybiz@inf.ug.edu.pl
}

Submitted: Feb 22, 2012; Accepted: Apr 12, 2012; Published: Apr 27, 2012

Mathematics Subject Classifications: 11B25

\begin{abstract}
A subsequence of the sequence $(1,2, \ldots, n)$ is called a 3 - $A P$-free sequence if it does not contain any three term arithmetic progression. By $r(n)$ we denote the length of the longest such $3-A P$-free sequence. The exact values of the function $r(n)$ were known, for $n \leqslant 27$ and $41 \leqslant n \leqslant 43$. In the present paper we determine, with a use of computer, the exact values, for all $n \leqslant 123$. The value $r(122)=32$ shows that the Szekeres' conjecture holds for $k=5$.
\end{abstract}

\section{Introduction}

Let $\langle n\rangle$ denote the sequence $(1,2, \ldots, n)$. A subsequence $\left(a_{1}, \ldots, a_{k}\right)$ of $\langle n\rangle$ is called a $3-A P$ free sequence if it does not contain any three elements $a_{p}, a_{q}, a_{r}$ such that $a_{q}-a_{p}=a_{r}-a_{q}$, or in other words if it does not contain any three term arithmetic progression. By $r(n)$ we denote the length of the longest $3-A P$-free sequences in $\langle n\rangle$. Sometimes (see [1]) the problem is equivalently presented in the set version. A subset $S \subset\{1,2, \ldots, n\}$ is called a 3 -AP-free if $a+c \neq 2 b$, for every distinct terms $a, b, c \in S$. The cardinality of the largest such subset $S$ is equal to $r(n)$.

The study of the function $r(n)$ was initiated by Erdős and Turán [2]. They determined the values $r(n)$, for $n \leqslant 23$ and $n=41$ (see Table 1 ), proved that $r(2 N) \leqslant N$, for $n \geqslant 8$, and conjectured that $\lim _{n \rightarrow \infty} r(n) / n=0$. This conjecture was proved in 1975 by Szemeredi [6]. Erdős and Turán also conjectured that $r(n)<n^{1-c}$, which was shown to be false by Salem and Spencer [4] who proved $r(n)>n^{1-c / \log \log n}$. The later result was improved by Behrend [1], who showed that $r(n)>n^{1-c / \sqrt{\log n}}$. The first non trivial upper bound was due by Roth [3] who proved $r(n)<\frac{c n}{\log \log n}$.

Recently Sharma [5] showed that Erdös and Turán gave the wrong value of $r(20)$ and determined the values of $r(n)$, for $n \leqslant 27$ and $41 \leqslant n \leqslant 43$ (see Tables 1 and 2). Erdös and Turán [2] noted that there is no three term arithmetic progression in the sequence of all numbers $n, 0 \leqslant n \leqslant \frac{1}{2}\left(3^{k}-1\right)$, which do not contain the digit 2 in the ternary scale. 
Hence, if we add 1 to each of these numbers, we obtain the 3 - $A P$-free sequence of length $2^{k}$ in $\left\langle\frac{1}{2}\left(3^{k}+1\right)\right\rangle$. Thus, we have

Lemma 1. $r\left(\frac{1}{2}\left(3^{k}+1\right)\right) \geqslant 2^{k}$, for every $k \geqslant 1$.

The Szekeres' conjecture (see [2]) says that $r\left(\frac{1}{2}\left(3^{k}+1\right)\right)=2^{k}$. The values given in $[2,5]$ (see Tables 1 and 2) show that the conjecture is true for $k \leqslant 4$. The Szekeres' conjecture has several interesting implications described in $[2,6]$. In the sequel we shall need the following lemmas from $[2,5]$.

Lemma 2. If $\left(a_{1}, \ldots, a_{k}\right)$ is a 3-AP=free sequence in $\langle n\rangle$ and $j<a_{1}$, then also $\left(a_{1}-\right.$ $\left.j, \ldots, a_{k}-j\right)$ is a 3 -AP-free sequence in $\langle n\rangle$.

Lemma 3. $r(n+m) \leqslant r(n)+r(m)$.

Lemma 4. $r(n) \leqslant r(n+1) \leqslant r(n)+1$.

If $r(n-1)<r(n)$ then we call $n$ a jump node. Whenever $r(n-2)<r(n-1)<r(n)$ we call $n$ a double jump.

Lemma 5. Three consecutive numbers cannot be jump nodes.

In this paper we determine, using computer, exact values of $r(n)$, for all $n \leqslant 123$. The value $r(122)=32$ shows that the Szekeres' conjecture holds for $k=5$. We also determined, for each jump node $n \leqslant 123, b(n)$ - the number of the longest 3 - $A P$-free sequences in $\langle n\rangle$, and an example of the longest 3 - $A P$-free sequence (see Tables 2 and 3 ).

\section{Algorithm}

In order to determine values of the function $r(n)$ we have designed a simple decision algorithm. This algorithm answers the question whether there is a 3 - $A P$-free sequence of length $k$ in $\langle n\rangle$. The algorithm uses the values $r(m)$, for $m<n$.

Algorithm 6. INPUT : $2 \leqslant k \leqslant n$ natural numbers

OUTPUT : YES if there exists $3-A P$-free sequence of length $k$ in $\langle n\rangle$; NO otherwise.

$1: a_{1}:=1 ; \quad a_{2}:=2 ; 1:=2$;

$2:$ APseq $:=\left(a_{1}, a_{2}\right)$

3 : repeat

4 : if APseq contains no 3-term arithmetic progression then

$5: \quad$ APseq $:=\left(a_{1}, a_{2}, \ldots, a_{l}, a_{l}+1\right)$

$6: \quad 1:=1+1$

$7: \quad$ else

8 : while $(1>1)$ and $\left(r\left(n-a_{l}\right)<\mathrm{k}-1+1\right)$

$9: \quad 1:=1-1$;

10: $\quad$ APseq $:=\left(a_{1}, a_{2}, \ldots, a_{l-1}, a_{l}+1\right)$

11: until $\left(a_{1} \neq 1\right)$ or $3-A P$-free sequence of length $\mathrm{k}$ is found

12: if $3-A P$-free sequence of length $\mathrm{k}$ is found, then return YES

13: if $\left(a_{1} \neq 1\right)$, then return NO 
The algorithm looks for a $3-A P$-free sequence of length $k$ in $\langle n\rangle$. It starts (lines 1-2) with the sequence $(1,2)$ of length $l=2$. At the beginning of each round of the "repeat until" loop (lines 3-11) the algorithm considers the sequence APseq $=\left(a_{1}, \ldots, a_{l-1}, a_{l}\right)$ of length $l \geqslant 2$. First, it checks if the sequence contains a 3-term arithmetic progression. If not, then it adds the element $a_{l}+1$ at the end of the sequence (lines 5-6). If APseq contains an arithmetic progression, then it looks for the next sequence to consider (lines $8-10)$. The first candidate is the sequence $\left(a_{1}, \ldots, a_{l-1}, a_{l}+1\right)$, but if $r\left(n-a_{l}\right)<k-l+1$, it is not possible to find remaining $k-l+1$ elements in the sequence $\left(a_{l}+1, \ldots, n\right)$ without arithmetic progression. In this case the algorithm sets $l:=l-1$ and checks if the sequence $\left(a_{1}, \ldots, a_{l-1}+1\right)$ will be good, and so on. The algorithm exits the main loop if it finds a $3-A P$-free sequence of the length $k$, or if $a_{1}>1$. In the later case it returns the answer NO, because, by Lemma 2, it suffices to consider sequences which start with 1 .

\section{Results}

We have used Algorithm 6 to determine the values of the function $r(n)$, for all $n \leqslant 120$. Observe that if we know the value $r(n)$, then we can obtain $r(n+1)$ after one application of the algorithm with the input $n+1$ and $k=r(n)+1$. All computations took about $30 \mathrm{~h}$ on personal computer. We have got the value $r(120)=30$. By Lemma $1, r(122) \geqslant 32$, thus by Lemma $4, r(121)=31$ and $r(122)=32$. Finally, by Lemma $5, r(123)=32$.

Tables 1, 2 and 3 present: the value of $r(n)$, the value of $b(n)$ - the number of the longest 3 - $A P$-free sequences, and an example of the longest 3 - $A P$-free sequence in $\langle n\rangle$, for each jump node $n \leqslant 123$. Table 1 contains the results obtained by Erdös and Turán [2] and by Sharma [5]. The values for $n<20$ were given by Erdős and Turán. They also gave

\begin{tabular}{|r|r|r|l|}
\hline$n$ & $r(n)$ & $b(n)$ & example of the longest 3- $A P$-free sequence \\
\hline \hline 1 & 1 & 1 & 1 \\
\hline 2 & 2 & 1 & 12 \\
\hline 4 & 3 & 2 & 124 \\
\hline 5 & 4 & 1 & 1245 \\
\hline 9 & 5 & 4 & 12489 \\
\hline 11 & 6 & 7 & 12451011 \\
\hline 13 & 7 & 6 & 1245101113 \\
\hline 14 & 8 & 1 & 124510111314 \\
\hline 20 & 9 & 2 & 1267914151820 \\
\hline 24 & 10 & 2 & 1257111618192324 \\
\hline 26 & 11 & 2 & 125711161819232426 \\
\hline
\end{tabular}

Table 1: Values of $r(n), b(n)$, and examples of the longest 3-AP-free sequences, for jump nodes $n \leqslant 26$, obtained in $[2,5]$. b(n) denotes the number of the longest 3-AP-free sequences in $\langle n\rangle$ 
the wrong value $r(20)=8$ and based on this value proved $r(41)=16$. Sharma gave the correct value of $r(20)=9$ and a new proof of $r(41)=16$. Our calculations confirm the values given by Erdos and Turan, for $n<20$, and those given by Sharma, for $20 \leqslant n \leqslant 27$ and $41 \leqslant n \leqslant 43$ (including the correction of the value $r(20)=9$ ). Tables 2 and 3 contain the results for $n \geqslant 30$. As we have mentioned above, the value $\mathrm{r}(41)=16$ was given in $[2,5]$, the others are new.

\begin{tabular}{|c|c|c|c|}
\hline$n$ & $r(n)$ & $b(n)$ & example of the longest $3-A P$-free sequence \\
\hline 30 & 12 & 1 & 13348911202223272830 \\
\hline 32 & 13 & 2 & 124891119222326283132 \\
\hline 36 & 14 & 2 & 12489132123262730323536 \\
\hline 40 & 15 & 20 & 12451011131428293132373840 \\
\hline 41 & 16 & 1 & 1245101113142829313237384041 \\
\hline 51 & 17 & 14 & 124510131417313537384046475051 \\
\hline 54 & 18 & 2 & 12561214151721313839424349515254 \\
\hline 58 & 19 & 2 & 1256121415172131383942434951525458 \\
\hline 63 & 20 & 2 & 125711161819242638394244485355566163 \\
\hline 71 & 21 & 4 & 12571017202226314146484953546364686971 \\
\hline 74 & 22 & 1 & 1279101420222325294650525355616566687374 \\
\hline 82 & 23 & 10 & 12489111922232628314957596263666871788182 \\
\hline
\end{tabular}

Table 2: Values of $r(n), b(n)$, and examples of the longest 3-AP-free sequences, for jump nodes $27 \leqslant n \leqslant 82$. The value $\mathrm{r}(41)=16$ was given in $[2,5]$.

\section{Discussion and open problems}

Sharma [5] asks if every $T_{k}=\frac{1}{2}\left(3^{k}+1\right)$ is a jump node. Our calculations and previous results show that, for $k \leqslant 5$, every $T_{k}$ is a double jump and there is only one longest 3 - $A P$-free sequence in $\left\langle T_{k}\right\rangle$, just the one described before Lemma 1 . We wonder if this is true for other $k$ ?

Using the values from Table 2 and 3 and a simple induction argument similar to the one described in [2] we can prove that $r(3 n) \leqslant n$, for every $n \geqslant 16$. Indeed, we know that $r(3 n) \leqslant n$, for every $16 \leqslant n \leqslant 31$, and $r(48)=16$. For $n \geqslant 32$, we have $r(3 n)=r(3(n-16)+48) \leqslant r(3(n-16))+r(48) \leqslant n-16+16=n$. 


\begin{tabular}{|c|c|c|c|}
\hline$n$ & $r(n)$ & $b(n)$ & example of the longest $3-A P$-free sequence \\
\hline 84 & $\overline{24}$ & 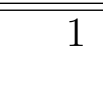 & $\begin{array}{l}134489161821222530374855606364676976 \\
77818284\end{array}$ \\
\hline 92 & 25 & 14 & $\begin{array}{l}126889131921222728395862646768717381 \\
8386879092\end{array}$ \\
\hline 95 & 26 & 8 & $\begin{array}{l}124510112223252631325556646567687677 \\
828391929495\end{array}$ \\
\hline 100 & 27 & 2 & $\begin{array}{l}136710122022252629313562666871727577 \\
858790919496100\end{array}$ \\
\hline 104 & 28 & 1 & 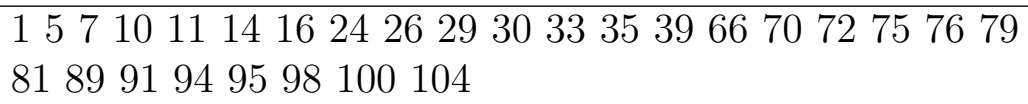 \\
\hline 111 & 29 & 6 & $\begin{array}{l}12561315192627303138424466 \quad 6872778081 \\
8489939599104107108111\end{array}$ \\
\hline 114 & 30 & 1 & 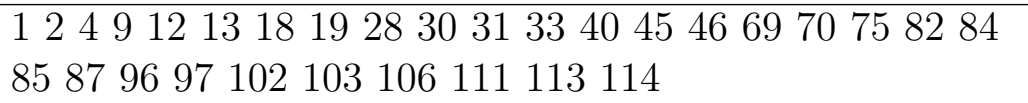 \\
\hline 121 & 31 & 70 & $\begin{array}{l}124510111314282931323738404182838586 \\
91929495109110112113118119121\end{array}$ \\
\hline 122 & 32 & 1 & $\begin{array}{l}124510111314282931323738404182838586 \\
91929495109110112113118119121122\end{array}$ \\
\hline
\end{tabular}

Table 3: Values of $r(n), b(n)$, and examples of the longest 3-AP-free sequences, for jump nodes $83 \leqslant n \leqslant 123$

\section{References}

[1] Behrend F.A. On Sets of Integers Which Contain No Three Terms in Arithmetic Progression. Proc. Nat. Acad. Sci. USA, 32:331-332, 1946.

[2] Erdős P., Turán P. On some sequences of integer. J. London Math. Soc., 11:261-264, 1936.

[3] Roth K. F. On certain set of integers. J. London Math. Soc., 28:104-109, 1953.

[4] Salem R., Spencer D. C. On Sets of Integers Which Contain No Three Terms in Arithmetic Progression. Proc. Nat. Acad. Sci. USA, 28:561-563, 1942.

[5] Sharma A. Sequences of Integers Avoiding 3-term Arithmetic Progressions. Elec. J. of Comb., 19:\#P27, 2012.

[6] Szemeredi E. On sets of integers containing no $k$ elements in arithmetic progression. Acta Arithmetica, 27:199-245, 1975. 